\title{
A Randomized Prospective Assessment of Safety and Effectiveness of Instillation of Tissue Plasminogen Activator in the Management of Intra-Abdominal Abscesses
}

\author{
Suhas A. Kotecha', ${ }^{1,2}$ Murray R. Asch3 ${ }^{\text {, Andrew Myers }}$, Dheeraj K. Rajan",2 \\ ${ }^{1}$ Department of Medical Imaging, Faculty of Medicine, University of Toronto, Toronto, ON, Canada \\ ${ }^{2}$ Division of Interventional Radiology, University Health Network, University of Toronto, Toronto, ON, Canada \\ ${ }^{3}$ Department of Radiology, Lakeridge Health, Oshawa, ON, Canada \\ Email: Dheeraj.rajan@uhn.ca
}

Received 22 June 2016; accepted 2 August 2016; published 5 August 2016

Copyright (C) 2016 by authors and Scientific Research Publishing Inc.

This work is licensed under the Creative Commons Attribution International License (CC BY).

http://creativecommons.org/licenses/by/4.0/

c) (i) Open Access

\begin{abstract}
Purpose: Catheter drainage of intra-abdominal abscesses has shown to be effective in reducing sepsis and mortality. We sought to determine whether or not tissue plasminogen activator (tPA), a fibrinolytic, is safe and facilitates resolution when used as an adjunct during drainage of intra-abdominal abscesses. Methods: A single-blinded, prospective, multi-center, randomized control trial was performed on patients presenting with image confirmed abscesses; the first group $(n=24)$ received catheter-directed tPA of their abscess and the second group $(n=23)$ had their abscess drained without tPA instillation. Variables examined included patient age, gender, location of the abscess, pre-drainage volumes and size of catheters inserted. Baseline variables were compared between tPA and non-tPA groups using a chi-square test for discrete variables and a Wilcoxon rank sum test for continuous variables and for the outcome of drainage volume. Results: Technical success of the drainage procedures was $100 \%$ for both groups. The tPA group had higher volume of drainage over the first three days (median for total after 3 days: without tPA: $300 \mathrm{~mL}$ vs. with tPA: $322 \mathrm{~mL}, \mathrm{p}=$ 0.42). At days 4 to 7 the tPA group had significant greater drainage volume than did the non-tPA patient group, $150 \mathrm{~mL}$ vs. $110 \mathrm{~mL}(p=0.046)$. The number of days to resume diet was also assessed in the 20 patients where this datum was available, 11 tPA and 9 non-tPA with no difference between groups. No adverse events, such as localized or systemic bleeding were encountered. Conclusion: Instillation of tPA into catheter directed intra-abdominal abscesses was safe, easy to perform and was associated with significantly improved drainage of abscesses from days 4 - 7.
\end{abstract}




\section{Keywords}

\section{Abscess, Catheter, Drainage, Hemorrhage}

\section{Introduction}

Previous work has demonstrated that fibrinolytics injected into abscesses are effective in shortening the treatment time and improving the clinical course for patients treated with percutaneous drainage techniques [1]-[3] and for treatment of malignant pleural effusions [4]. One study determined that tPA was also effective and safe in the drainage of complicated pediatric parapneumonic effusions [5]. Based on the results reported in these studies, we studied the clinical efficacy and safety of tissue plasminogen activator (tPA) in the drainage of intra-abdominal abscesses.

Three randomized studies have been published of which only one was published before initiation of this study. This study utilized urokinase, not tPA, for intra-abdominal abscesses [2]. The study did not evaluate flow rates per day. Another randomized control trial demonstrated that loculated abdominal and pelvic abscesses demonstrated improved drainage with a three-day course of twice daily alteplase infusion [3]. Sample size calculation required 80 patients enrollment with twenty patients eventually enrolled and 11 receiving tPA. Daily flow rates were not assessed and study sample size was not achieved. The remaining study assessed use of tPA for appendiceal abscesses in children with the authors concluding that there was no clinical benefit to use fibrinolytics administered into appendiceal abscesses [6].

The objective of this study was to evaluate the clinical efficacy and safety of intra-abdominal instillation of tPA in patients who required abscess tube drainage for intra-abdominal abscesses. We aimed to build on previous reports, by designing a simple and safe method for delivery of intra-cavitary tPA. This prospective randomized study used a simple once a day instillation of tPA for three days with the concentration of tPA dependent upon the initial volume of the abscess drained.

\section{Materials and Methods}

\subsection{Patients}

Institutional review board and Health Canada approval for off label study of tPA was obtained for this study. All patients included in the study signed informed consent. This was a multi-center single blinded prospective randomized study conducted from June 2005 to December 2013 at three tertiary care and one community medical centers (NCT00417768).

All patients found to have complex fluid collections that met imaging criteria of abscesses at the time of cross sectional imaging studies (ultrasound, and/or, CT, and/or, MRI) who presented to the interventional radiology for percutaneous image guided drainage and were considered for entry into the study. Inclusion and exclusion criteria are presented in Table 1. Causes for early termination in participation of the study are presented in Table 2.

Our outcome measures were total volume of fluid drained, daily drainage volume, number and type of interventions after the first drainage procedure, length of stay in hospital after first drain, time to change in diet and imaging resolution. Outcome measures were evaluated by a dedicated independent research coordinator.

\subsection{Methods}

Patients underwent percutaneous drainage using standard image guided techniques by fellowship trained interventional radiologists. Tube sizes placed ranged from 10 French to 14 French. Patients in the study arm received once daily instillations tissue plasminogen activator (tPA) solution volume of $25 \%$ of the initial drained abscess volume for three days for a total of three treatments. The concentration of tissue plasminogen activator (tPA) administered was $0.3 \mathrm{mg}$ of $\mathrm{tPA} / \mathrm{mL}$ (6 mg of Cathflo reconstituded with sterile water (Hoffmann-La Roche, Mississauga, Ontario)) mixed in $0.9 \% 20 \mathrm{~mL}$ of sterile normal saline instilled into the abscess with a maximum volume of $100 \mathrm{~mL} /$ day (30 mg/day). The catheter was then cleared following administration of the tPA solution with $5 \mathrm{~mL}$ of sterile normal saline by the physician who performed the original drainage procedure. A sham procedure of examining the drainage tube daily and pretending to inject a solution, under the cover of a bedsheet 
Table 1. Inclusion criteria and exclusion criteria.

\section{Exclusion Criteria}

- Ability to consent to the procedure

- Age 18 - 80

- Intra-abdominal fluid collection thought to be an abscess which when a drain was inserted, returned purulent fluid and that has not been previously percutaneously drained but requires percutaneous drainage.
- Inappropriate antibiotic coverage prior to the drainage procedure

- Pregnancy or lactating

- Child bearing potential who is not using a reliable method of contraception

- Previous allergic reaction to TPA

- Pancreatic pseudocysts and collections thought to be hematomas

- Inability or unwillingness to consent to the procedure

- Treatment with any investigational drug or investigational biologic within 30 days of initiating study treatment

- Scheduled major surgery during the study period

- Concomitant administration of unfractionated heparin intravenously for the purpose of systemic anticoagulation

- Patients with a baseline platelet count $<50,000 /$ ul, INR $>1.6$ (when not corrected with fresh frozen plasma at the time of the procedure)

- Known coagulopathy, systemic therapeutic anticoagulation or any condition in which bleeding constitutes a significant hazard or would be particularly difficult to manage because of its location, or who are at high risk for embolic complications (e.g., recent pulmonary embolism, deep vein thrombosis, endarterectomy)

- Major hemorrhage within the previous 4 weeks, defined as bleeding resulting in a drop in hemoglobin of greater than $20 \mathrm{~g} / \mathrm{L}$ or bleeding requiring transfusion of packed red blood cells with other clinical evidence or suspicion of bleeding

- History of intracranial hemorrhage within the 4 weeks prior to drainagee prior to the drainage procedure

- Child bearing potential who is not using a reliable method of contraception

- Previous allergic reaction to TPA

- Pancreatic pseudocysts and collections thought to be hematomas

- Inability or unwillingness to consent to the procedure

- Treatment with any investigational drug or investigational biologic within 30 days of initiating study treatment

- Scheduled major surgery during the study period

- Concomitant administration of unfractionated heparin intravenously for the purpose of systemic anticoagulation

- Patients with a baseline platelet count $<50,000 /$ ul, INR $>1.6$ (when not

corrected with fresh frozen plasma at the time of the procedure)

- Known coagulopathy, systemic therapeutic anticoagulation or any condition in which bleeding constitutes a significant hazard or would be particularly difficult to manage because of its location, or who are at high risk for embolic complications (e.g., recent pulmonary embolism, deep vein thrombosis, endarterectomy)

- Major hemorrhage within the previous 4 weeks, defined as bleeding resulting in a drop in hemoglobin of greater than $20 \mathrm{~g} / \mathrm{L}$ or bleeding requiring transfusion of packed red blood cells with other clinical evidence or suspicion of bleeding - History of intracranial hemorrhage within the 4 weeks prior to drainage

was performed for patients randomized to not receive tPA in order to blind the patient and the patient's primary care physician to the type of treatment the patient was receiving. This was performed after initial attempted complete aspiration of the intraabdominal collection. Dwell time of the tPA solution was 1 hour prior to reinitiation of gravity drainage. All tubes from both groups were subsequently flushed with $5 \mathrm{~mL}$ normal saline twice a day. Patients were followed when possible to 30 days post resolution of the abscess in order to assess for potential differences in recurrences in the two treatment groups.

\subsection{Rationale for tPA Dose}

Meta-analysis of randomized comparisons of urokinase and tPA as full-dose thrombolytic agents for arterial use suggested that $1 \mathrm{mg}$ tPA was likely equivalent in thrombolytic potency to 36,000 units urokinase [7] whereas for venous catheter clearance an equivalent of $5000 \mathrm{U}$ urokinase to $1 \mathrm{mg}$ tPA has been suggested based on prior studies [7]. In a randomized study using urokinase to facilitate abscess drainage [2], 12,500 $\mathrm{U}$ of urokinase or equiv. of 1 - $2.5 \mathrm{mg}$ tPA was used for $1-5 \mathrm{~cm}$ diameter abscesses, 5 - $10 \mathrm{~cm}$ size, 50,000 $\mathrm{U}$ of urokinase or the equivalent of $2-10 \mathrm{mg}$ of tPA was used and for abscesses $>10 \mathrm{~cm} \mathrm{100,000} \mathrm{U}$ of urokinase was used or the equivalent of 4 - $20 \mathrm{mg}$ of tPA. 
Table 2. Protocol termination and patient withdrawal rules.

1) Patient refusal or non-compliance to continue with the trial

2) Patient undergoes surgical intervention related to the abscess during the study period

3) Patient suffers a serious adverse event as a result of the study

- drop in hemoglobin greater than $20 \mathrm{~g} / \mathrm{L}$

- bleeding requiring transfusion

- allergic reaction requiring medical intervention

4) Findings observed during the study lead to early termination either due to clear benefit or disadvantages of therapy

5) Imaging findings demonstrate worsening of the abscess either as dissemination or increase in volume $>25 \%$

6) White blood cell count remains elevated above the baseline recorded value 48 hours beyond intervention

7) The drainage catheter becomes dislodged from the abscess cavity (determined by routine imaging examinations)

8) Technical malfunction of the catheter during drug administration (fracture or hole in the catheter or the catheter is occluded)

Using the volume formula of $(\pi \times$ length $\times$ width $\times$ depth $) / 6$ for volume of the abscess (pre and post drainage) assuming a spherical shape $1-5 \mathrm{~cm}$ has a maximum volume of approximately $62 \mathrm{cc}$ for $4.5 \mathrm{mg}$ of tPA, $5-10$ $\mathrm{cm}$ has a volume of $62-500 \mathrm{cc}$ for $4.5 \mathrm{mg}-30 \mathrm{mg}$ dose, $>10 \mathrm{~cm}$ has a volume of $>500 \mathrm{cc}$ for $30 \mathrm{mg}$ dose. We chose to use $25 \%$ of the initial drained volume as to prevent over distension of the abdominal cavity.

We arbitrarily roughly doubled the extrapolated dose of tPA and delivered this dose three times as this was a pilot study given that it is unknown what dose will be effective for resolution of abscesses. Rather than using a potentially ineffective dose, we have elected to use the higher dose to determine efficacy and safety. Future studies may elucidate a lower effective dose but this cannot be investigated until the treatment proposed in this study was proven to be clinically effective, safe and cost effective.

\subsection{Statistical Methods and Definitions}

Randomization was performed using computer-generated random numbers in blocks of 10 with one to one randomization. Randomization codes of either tPA or no tPA were placed in sequentially numbered, opaque, sealed envelopes at each treatment site. Once a patient was recruited and consented, the next numbered envelope was opened by the operator who had no knowledge of the randomization code just prior to percutaneous drainage. While the operator was not blinded to the treatment group, they were blinded to the results over the course of the study.

The study sample size was determined based on the results of a prior randomized study that examined use of intracavitary urokinase on length of stay, days of drainage and febrile course [2]. Significant differences in length of stay and cost were observed with 42 patients randomized. Based off these results, a conservative patient population size calculation for this study was 100 patients with 50 patients to be randomized to each arm with a confidence interval $>95 \%$ obtained with a power $>80 \%$.

The location of the intra-peritoneal abscess, most commonly determined by a diagnostic CT scan prior to intervention, was categorized as right upper quadrant, right lower quadrant, left lower quadrant, pelvic and "intra-peritoneal" when the abscess traversed more than one quadrant. While all cases were intra-peritoneal in location, the descriptor "intra-peritoneal" was used when the location of the abscess did not conveniently fit into a quadrant descriptor or the size of the abscess was large enough to involve multiple quadrants or crossed over into more than one quadrant.

Descriptive statistics for discrete baseline data are reported as number and percentage and for continuous variables reported as median and range. The drainage volume data has a non-normal distribution, in most cases the standard deviation exceeded the mean and therefore the median was reported and non-parametric tests were used. Baseline variables were compared between tPA and non-tPA groups using a chi-square test for discrete variables and a Wilcoxon rank sum test for continuous variables and for the outcome of drainage volume. Additionally, drainage volume was also compared between treatment groups in a model including age and gender. This was done replacing the drainage volume with ranked volume, and using analysis of variance (ANOVA) on the 
ranked data. The alpha-level was set at 0.05 for statistical significance. All analyses were done using SAS version 9.4 (Cary, NC).

Patients were followed for as long as data was reliably available per standard of practice. For the non-tPA group of patients, tube residence was followed for up to 50 days; for the tPA group of patients, tube residence was followed for up to 124 days. However, we restricted our analysis for the first seven days as beyond this time we did not have enough patients for reliable statistical comparison.

\section{Results}

Total enrollment was 47 patients who met criteria for inclusion into the study with 23 patients within the sham group (no tPA) and 24 patients for the tPA group. There was no significant difference between groups pre drainage for assessed patient and abscess variables including volume of the abscesses pre-drainage (Table 3).

Overall 32 (68\%) of the patients were male with $75 \%$ and $61 \%$ in the tPA and non-tPA groups respectively (p = 0.36). "Intra-peritoneal" was the most common location of the abscess in the tPA group, occurring in 12 (50\%) of patients, whereas the most common location in the non-tPA group was in the left lower quadrant, occurring in 9 cases (39.1\%), closely followed by intra-peritoneal (8 cases, 34.8\%). There were 5 cases (20.8\%) in the right lower quadrant and 2 right upper quadrant cases (8.3\%) that received tPA. Similarly, in the non-tPA group there were 3 cases (13\%) in the right upper quadrant and right lower quadrant, respectively. Only a single case (4.2\%) was classified as a pelvic abscess, and this case was treated with tPA. There was a single case in the non-tPA group and in the tPA group in which there was prior tube drainage. However, as per the exclusion criteria for the

Table 3. Patient variables pre-drainage.

\begin{tabular}{|c|c|c|c|}
\hline & Sham $(n=23)$ & $\mathrm{tPA}(\mathrm{n}=24)$ & P-value \\
\hline Age, mean (SD) & $66.9(14.7)$ & $59.9(17.4)$ & $0.15^{1}$ \\
\hline Male gender, n (\%) & $14(60.9 \%)$ & $18(75.0 \%)$ & $0.36^{2}$ \\
\hline \multicolumn{4}{|l|}{ Diagnosis location, n (\%) } \\
\hline RUG & $3(13.0 \%)$ & $2(8.3 \%)$ & \multirow{5}{*}{$0.34^{2}$} \\
\hline RLQ & $3(13.0 \%)$ & $5(20.8 \%)$ & \\
\hline Pelvic & $0(0.0 \%)$ & $1(4.2 \%)$ & \\
\hline LLQ & $9(39.1 \%)$ & $4(16.7 \%)$ & \\
\hline Intra-peritoneal & $8(34.8 \%)$ & $12(50.0 \%)$ & \\
\hline Previous Drain Tube, n (\%) & $1(4.3 \%)$ & $1(4.2 \%)$ & $1.0^{1}$ \\
\hline Pre-drain volume (cc), median (range) & $115(61,000)$ & $95(51,200)$ & $0.38^{3}$ \\
\hline Hg, mean $(\mathrm{SD})^{\mathrm{a}}$ & $108.6(16.6)$ & $113.0(18.9)$ & $0.41^{1}$ \\
\hline Platelets, median (range) ${ }^{\mathrm{a}}$ & $412(60,782)$ & $375(134,782)$ & $0.72^{3}$ \\
\hline Days to resume diet, median (range) ${ }^{\mathrm{b}}$ & $1(0.7)$ & $0(0.4)$ & $0.33^{3}$ \\
\hline \multicolumn{4}{|l|}{ Postop days to $\mathrm{TPN}^{\mathrm{c}}$} \\
\hline$=0$ (same day as surgery) & $10(76.9 \%)$ & $10(76.9 \%)$ & $1.0^{2}$ \\
\hline$>0$ & $3(23.1 \%)$ & $3(23.1 \%)$ & \\
\hline \multicolumn{4}{|l|}{ Tube size, n (\%) } \\
\hline $8 \mathrm{~F}$ & $1(4.4 \%)$ & $1(4.2 \%)$ & \\
\hline $10 \mathrm{~F}$ & $10(43.5 \%)$ & $7(29.2 \%)$ & $0.63^{2}$ \\
\hline $12 \mathrm{~F}$ & $11(47.8 \%)$ & $16(66.7 \%)$ & \\
\hline $14 \mathrm{~F}$ & $1(4.4 \%)$ & $0(0.0 \%)$ & \\
\hline
\end{tabular}

${ }^{\mathrm{a}} 1$ patient in tPA missing data. ${ }^{\mathrm{b}} 14$ and 15 patients missing data in Sham and tPA groups respectively. ${ }^{\mathrm{c}} 10$ and 11 patients missing data in Sham and tPA groups respectively. ${ }^{1}$ Two-sample test; ${ }^{2}$ Fisher's exact test; ${ }^{3}$ Wilcoxon rank sum test. 
study, no cases would be considered if there was prior tube drainage of the intra-peritoneal abscess that was previously enrolled in the study.

Technical success of the drainage procedures was $100 \%$ for both groups. The median volume drained in the tpA group exceeded that in the non-tPA group on each of the first 7 days, though the difference in volume was not always statistically significant (Table 4). The median daily volume drained was highest on day one and similar for both groups (medians of 109.5 and 107.5 in the tpA and non-tpA groups respectively, $\mathrm{p}=0.84$ ). The group that received intra-abscess tPA had higher volume of drainage over the first three days (median for total after 3 days: without-tPA: $300 \mathrm{~mL}$ vs. with tPA: $322 \mathrm{~mL}, \mathrm{p}=0.42$ ) and on day 3 specifically ( $50 \mathrm{~mL}$ versus 115 $\mathrm{mL}$ for the tPA group, $\mathrm{p}=0.02$ ). Looking at days 4 to 7 the tPA group had a borderline significant greater drainage volume than did the non-tPA patient group, $150 \mathrm{~mL}$ vs. $110 \mathrm{~mL}, \mathrm{p}=0.046$. Finally looking at the total drainage volume over the first seven days the median was marginally higher in the tPA patients, $465 \mathrm{~mL}$ vs. 452 $\mathrm{mL}$ but not significant $(\mathrm{p}=0.23)$. Looking at the statistical models including age and gender in addition to group status yielded consistent results with those described above (Table 4).

The number of days to resume diet was also assessed in the 20 patients where this data was available ( $11 \mathrm{tPA}$ and 9 non-tPA). While most patients in this study were nil per os (NPO) at the time of tube insertion there were patients who were maintained on clear diets. As such, a change in diet (i.e., from NPO to clear fluids or diet as tolerated) was considered as "resuming diet". The median number of days to "resume diet" in the sham group was 0.0 and 1.0 in the tPA group, $\mathrm{p}=0.33$.

Imaging resolution of the abscesses could not be ascertained as all drains were removed by referring clinicians based on clinical resolution of symptoms and drain output $<10 \mathrm{~mL} /$ day. There were no major or minor complications observed including intra-abdominal hemorrhage requiring clinical intervention, sepsis or allergic reactions.

\section{Discussion}

Catheter directed abscess drainage has been shown to be effective in reducing morbidity and mortality but can be particularly difficult to drain if the collection is thick or septated. In a rat model of intra-peritoneal abscess [8], and empyemas [9] tPA has been shown to be effective in improving drainage of abscesses. A recent retrospective review in children with thick or septated intra-abdominal abscess demonstrated that tPA was safe in their management and possibly reduced hospital stay [10].

In a retrospective study by Beland, 46 abdominal and pelvic abscesses that were refractory to routine drainage underwent administration of $4-6 \mathrm{mg}$ of tPA twice daily for three days. The tPA dose was mixed with 25 cc of normal saline and administered a mean of 6.4 days after initial drainage. The authors found that tPA administration

Table 4. Drainage volumes between groups.

\begin{tabular}{ccccccc}
\hline & \multicolumn{2}{c}{ Non tPA $(\mathrm{n}=23)$} & & tPA $(\mathrm{n}=24)$ & Wilcoxon Rank Sum Test & ANOVA of ranked data \\
\hline & $\mathrm{N}$ & Median $(\mathrm{IQR})$ & $\mathrm{N}$ & Median $(\mathrm{IQR})$ & P-value & P-value $^{\mathrm{A}}$ \\
\hline Day 1 & 22 & $\mathbf{1 0 7 . 5}(40-160)$ & 24 & $\mathbf{1 0 9 . 5}(45-175)$ & $\mathbf{0 . 8 4}$ & $\mathbf{0 . 5 2}$ \\
Day 2 & 23 & $\mathbf{9 0 . 0}(20-160)$ & 23 & $\mathbf{1 4 0 . 0}(30-200)$ & $\mathbf{0 . 1 9}$ & $\mathbf{0 . 0 8}$ \\
Day 3 & 23 & $\mathbf{5 0 . 0}(16-80)$ & 23 & $\mathbf{1 1 5 . 0}(25-157)$ & $\mathbf{0 . 0 4}$ & $\mathbf{0 . 0 2}$ \\
Day 4 & 21 & $\mathbf{3 6}(20-75)$ & 23 & $\mathbf{7 5 . 0}(20-130)$ & $\mathbf{0 . 0 7}$ & $\mathbf{0 . 0 6}$ \\
Day 5 & 21 & $\mathbf{1 0 . 0}(1-40)$ & 22 & $\mathbf{2 1 . 0}(5-120)$ & $\mathbf{0 . 1 2}$ & $\mathbf{0 . 0 3}$ \\
Day 6 & 20 & $\mathbf{1 5 . 0}(1-42.5)$ & 22 & $\mathbf{3 4 . 0}(5-120)$ & $\mathbf{0 . 0 7}$ & $\mathbf{0 . 0 1}$ \\
Day 7 & 20 & $10(1-15)$ & 21 & $\mathbf{3 0 . 0}(10-60)$ & $\mathbf{0 . 0 1}$ & $\mathbf{0 . 4 2}$ \\
Sum Days 1 - 3 & 22 & $\mathbf{3 0 0}(116-425)$ & 23 & $\mathbf{3 2 2 . 0}(114-528)$ & $\mathbf{0 . 0 4 6}$ & $\mathbf{0 . 0 2}$ \\
Sum Days 4 - 7 & 20 & $\mathbf{1 1 0 . 0}(25-167)$ & 21 & $\mathbf{1 5 0 . 0}(44-365)$ & $\mathbf{0 . 2 3}$ & $\mathbf{0 . 0 8}$ \\
Sum Days 1 - 7 & 19 & $\mathbf{4 5 2 . 0}(157-635)$ & 21 & $\mathbf{4 6 5 . 0}(192-946)$ & \\
\hline
\end{tabular}


resulted in high effectiveness with no local bleeding complications [1]. A comparison to abscesses not treated with tPA was not performed and daily drainage volumes were not assessed.

Three randomized studies have been published. The first utilized urokinase where 42 patients with intra-abdominal abscesses were randomized based on culture positive results or clinical suspicion of an abscess. Size dependent doses of urokinase were injected into abscesses at 8 hour intervals for 4 days compared to saline in the placebo group. Length of stay and treatment cost were significantly less with the urokinase group with no difference in tube residence time [2]. The study did not evaluate flow rates per day as our study did and clinical practice of keeping patients within the hospital while having abscess tubes in place has likely changed within the last 15 years. However, this was the first randomized study conducted where fibrinolytics were used for abscesses and was therefore used as the basis of our sample size determination.

The second prospective randomized control trial demonstrated that loculated abdominal and pelvic abscesses demonstrated improved drainage with a three day course of twice daily alteplase infusion [3]. Either $2 \mathrm{mg}$ or 4 mg of tPA mixed with saline and delivered as half the volume of the abscess was administered twice daily for three days with success defined as removal of the catheter within 30 days. Sample size calculation required 80 patient enrollment with twenty patients eventually enrolled and 11 receiving tPA. Thirty day success was $73 \%$ in the tPA group compared to $22 \%$ in the saline group [3]. Daily flow rates were not assessed and study sample size as in our study was not achieved.

In a randomized study assessing use of tPA for appendiceal abscesses in children with 62 patients randomized to a fixed dose of twice daily instillation of $13 \mathrm{~mL} 10 \%$ tPA or $13 \mathrm{~mL}$ normal saline. There was a trend towards more drainage in the tPA group however this group had a significantly longer duration of hospital stay. The increased drainage resulted in longer hospital stay as this was performed in children. The authors concluded that there was no clinical benefit to use of fibrinolytics administered into appendiceal abscesses [6].

Compared to these studies, with the exception of one, we adjusted dose of fibrinolytic and the volume it was delivered in based on the pre-drainage abscess volume size. Unlike other studies, daily flow rates were assessed and within the adult population we attained a relatively large sample size. Another strength of this study is that a prospective, randomized study was performed. Although we did not attain the study sample size estimation, a significant difference in drainage volumes was observed on day 3 and from days 4 - 7 although no difference in change in diet was observed. The procedure was straight forward and easy to complete. It required a once a day instillation of tPA via a catheter for three days. This differs from previous studies in which twice a day instillation of a fibrinolytic was performed [1], [3], [6]. In this study the initial drainage involved the infusion of tPA. The volume of tPA instilled was similar to that used by [3], and was directly related to the size of the abscess at the time of initial drainage.

Given the improved drainage with tPA, this raises the possibility that there may be additional benefit with a higher concentration of tPA or for infusion for greater than 3 days. Alternatively, effective dose may be lower than used in this study. Additional studies are required to address the lowest possible effective dose and if improved drainage does result in improved clinical outcomes and cost reduction. A limitation with the current methodology is that the volume initially drained, which is used to determine the concentration of tPA instilled, may under estimate the actual abscess if the abscess was particularly viscous or loculated.

The obvious weakness of our study is that we did not complete recruitment to the predetermined sample size. The failure to recruit was multi-factorial over an 8 year span and given our low recruitment rate and ongoing costs, we decided to terminate the study before full sample size was attained. At the primary study hospital, surgical practice became much more specialized resulting in many patients being excluded based on risk of hemorrhage and multiple prior or expected surgeries. In addition, another initial goal set out was to quantify whether catheter dwell time was reduced in the tPA arm. However, in an evolution of patient care over time where patients were originally kept in hospital until there were signs of abscess resolution, the majority of the patients enrolled in this study were discharged from hospital within 24 hours with the catheter in situ and received daily home care. We could not reliably account for when the catheter was removed in the majority of our patients beyond seven days and if imaging resolution occurred. This also limited our ability to address whether tPA instillation decreased treatment costs. However, a previous phase II study has shown instillation of an intra-cavitary fibrinolytic significantly reduces length of hospital stay and treatment costs [2].

An additional limitation of this study was that all abscesses drained were considered to be equivalent. Studies have demonstrated that a multi-loculated, highly viscous abscess is more difficult to drain than an abscess that is unilocular and has lower fluid viscosity [1], [11]. Given that tPA acts to break down fibrin clots, it is likely that 
tPA would have an added benefit for multi-loculated, viscous abscesses compared to a "simple” abscess. Future studies could address this issue by qualitatively categorizing the abscess as simple versus complicated at the time of initial drainage or even at the time of diagnostic CT scan or ultrasound. Finally, we could only assess a change in diet in twenty patients limiting any meaningful interpretation.

Although the aforementioned studies including our results all point to efficacy of fibrinolytics at improving abscess drainage, there is variation in dose, method of dosing and duration of administration. More prospective randomized studies are likely needed before fibrinolytic use and the associated increased cost becomes part of routine percutaneous abscess drainage. Also, addition of recombinant deoxyribonuclease with fibrinolytics may also improve on drainage over fibrinolytics alone as observed in a study where complex pleural empyemas were successfully drained in $92 \%$ of patients [12].

\section{Conclusion}

In conclusion, this study supports the safety and efficacy of intra-cavitary tissue plasminogen activator to facilitate drainage rates of abscesses with a significant difference observed compared to no fibrinolytic used. Future studies that evaluate the effectiveness of different concentrations of the tPA may be useful.

\section{References}

[1] Beland, M.D., Gervais, D.A., Levis, D.A., Hahn, P.F., Arellano, R.S. and Mueller, P.R. (2008) Complex Abdominal and Pelvic Abscesses: Efficacy of Adjunctive Tissue-Type Plasminogen Activator for Drainage. Radiology, 247, 567573. http://dx.doi.org/10.1148/radiol.2472070761

[2] Haaga, J.R., Nakamoto, D., Stellato, T., et al. (2000) Intracavitary Urokinase for Enhancement of Percutaneous Abscess Drainage: Phase II Trial. American Journal of Roentgenology, 174, 1681-1685. http://dx.doi.org/10.2214/ajr.174.6.1741681

[3] Cheng, D., Nagata, K.T. and Yoon, H.C. (2008) Randomized Prospective Comparison of Alteplase versus Saline Solution for the Percutaneous Treatment of Loculated Abdominopelvic Abscesses. Journal of Vascular and Interventional Radiology, 19, 906-911. http://dx.doi.org/10.1016/j.jvir.2008.03.007

[4] Gilkeson, R.C., Silverman, P. and Haaga, J.R. (1999) Using Urokinase to Treat Malignant Pleural Effusions. American Journal of Roentgenology, 173, 781-783. http://dx.doi.org/10.2214/ajr.173.3.10470923

[5] Weinstein, M., Restrepo, R., Chait, P.G., Connolly, B., Temple, M. and Macarthur, C. (2004) Effectiveness and Safety of Tissue Plasminogen Activator in the Management of Complicated Parapneumonic Effusions. Pediatrics, 113, e182185. http://dx.doi.org/10.1542/peds.113.3.e182

[6] St Peter, S.D., Shah, S.R., Adibe, O.O., et al. (2015) Saline vs Tissue Plasminogen Activator Irrigations after Drain Placement for Appendicitis-Associated Abscess: A Prospective Randomized Trial. Journal of the American College of Surgeons, 221, 390-396. http://dx.doi.org/10.1016/j.jamcollsurg.2015.03.043

[7] Clase, C.M., Crowther, M.A., Ingram, A.J. and Cina, C.S. (2001) Thrombolysis for Restoration of Patency to Haemodialysis Central Venous Catheters: A Systematic Review. Journal of Thrombosis and Thrombolysis, 11, 127-136. http://dx.doi.org/10.1023/A:1011272632286

[8] van Goor, H., de Graaf, J.S., Kooi, K., et al. (1994) Effect of Recombinant Tissue Plasminogen Activator on IntraAbdominal Abscess Formation in Rats with Generalized Peritonitis. Journal of the American College of Surgeons, 179, 407-411.

[9] Ray, T.L., Berkenbosch, J.W., Russo, P. and Tobias, J.D. (2004) Tissue Plasminogen Activator as an Adjuvant Therapy for Pleural Empyema in Pediatric Patients. Journal of Intensive Care Medicine, 19, 44-50. http://dx.doi.org/10.1177/0885066603259942

[10] Shawyer, A.C., Amaral, J.G. and Langer, J.C. (2012) The Role of Tissue Plasminogen Activator in the Management of Complex Intra-Abdominal Abscesses in Children. Journal of Pediatric Surgery, 47, 1380-1384. http://dx.doi.org/10.1016/j.jpedsurg.2011.12.006

[11] Park, J.K., Kraus, F.C. and Haaga, J.R. (1993) Fluid Flow during Percutaneous Drainage Procedures: An in Vitro Study of the Effects of Fluid Viscosity, Catheter Size, and Adjunctive Urokinase. American Journal of Roentgenology, 160, 165-169. http://dx.doi.org/10.2214/ajr.160.1.8416618

[12] Piccolo, F., Pitman, N., Bhatnagar, R., et al. (2014) Intrapleural Tissue Plasminogen Activator and Deoxyribonuclease for Pleural Infection. An Effective and Safe Alternative to Surgery. Annals of the American Thoracic Society, 11, 1419-1425. http://dx.doi.org/10.1513/AnnalsATS.201407-3290C 


\section{Submit or recommend next manuscript to SCIRP and we will provide best service for you:}

Accepting pre-submission inquiries through Email, Facebook, LinkedIn, Twitter, etc.

A wide selection of journals (inclusive of 9 subjects, more than 200 journals)

Providing 24-hour high-quality service

User-friendly online submission system

Fair and swift peer-review system

Efficient typesetting and proofreading procedure

Display of the result of downloads and visits, as well as the number of cited articles

Maximum dissemination of your research work

Submit your manuscript at: http://papersubmission.scirp.org/ 